\title{
A photo-distributed rash with neurological problems in an elderly man: Don't forget pellagra!
}

\section{Sara Dahhouki, Aicha Harouni Alaoui, Khadija Issoual, Zakia Douhi, Sara Elloudi, Hanane Baybay, Salim Gallouj, Fatima Zahra Mernissi}

\author{
Department of Dermatology, Hassan II Hospital University, Fez, Morocco
}

Corresponding author: Dr. Sara Dahhouki, E-mail: dahhoukisara@gmail.com

\begin{abstract}
Pellagra is caused by deficiency of niacin or its amino acid precursor tryptophan. Clinical features are characterized by dermatitis, dementia and diarrhea. Dermatitis is observed only in $33 \%$ of patients. We report a 110-year-old woman, with history of diarrhea and dementia, who presented a bilateral and symmetrical dermatosis, characterized by welldemarcated violaceous plaques due to sun exposition. Dermatological symptoms had directed investigations. Pellagra was diagnosed and treatment with supplement and oral niacin was started, with significant improvement.
\end{abstract}

Key words: Niacin deficiency; Dermatitis; Dementia; Diarrhea; Oral niacin; Vitamin supplementation

\section{INTRODUCTION}

Pellagra is a rare systemic disorder due to niacin deficiency (vitamin B3 or PP). It associates a photo-induced skin rash, transit disorders, and in case of prolonged deficiency, neurological signs. The most common etiology is lack of intake, but other causes may be involved [1]. Here we report a case of A 110-year-old woman with pellagra.

\section{CASE REPORT}

A 110-year-old woman, with recent history of dementia and intermittent diarrhea, presented with recurrence of a painful lilaceous eruption, 3 months earlier, on the back of legs, due to sun exposure of this area. On examination, patient was confused. Dermatological examination revealed well-defined violaceous plaques with erythematous margins and large area of blistering on her legs with surrounding hyperpigmentation (Figs. la - ld). Neurological examination noticed a spastic weakness with power of $4 / 5$ in all the four limbs with rigidity at both the wrist joints. The deep tendon reflexes were bilaterally brisk. Abdominal examination was normal. Directed investigations objected an anemia with low levels of calcium, phosphorus and magnesium. There were no signs of chronic liver disease. Serum niacin level estimation was conducted, which was low. The diagnosis retained was pellagra and treated with niacin $300 \mathrm{mg}$ a day, and vitamin supplementation with hyperprotidic diet, were started. We noted a significant improvement of dermatological, neurological and digestive symptoms at 2 weeks.

\section{DISCUSSION}

Pellagra is a rare deficiency disease. Our observation illustrates the importance of highlighting the diagnosis of pellagra when we have an association of a cutaneous eruption due to sun exposition, digestive signs and neurological manifestations [2]. Niacin deficiency may be related to a malabsorption syndrome or intake deficiency, chronic ethylism and certain drugs such as 5-fluorouracil and phenobarbital are also implicated [3]. Skin symptoms are usually the first to appear. Digestive symptoms occur secondary and are a sign of the severity of the disease. Neurological

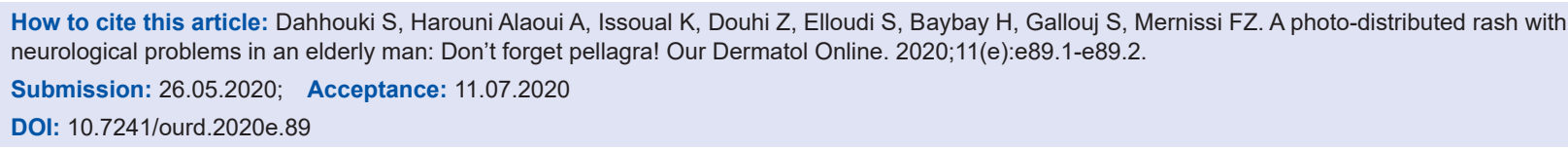



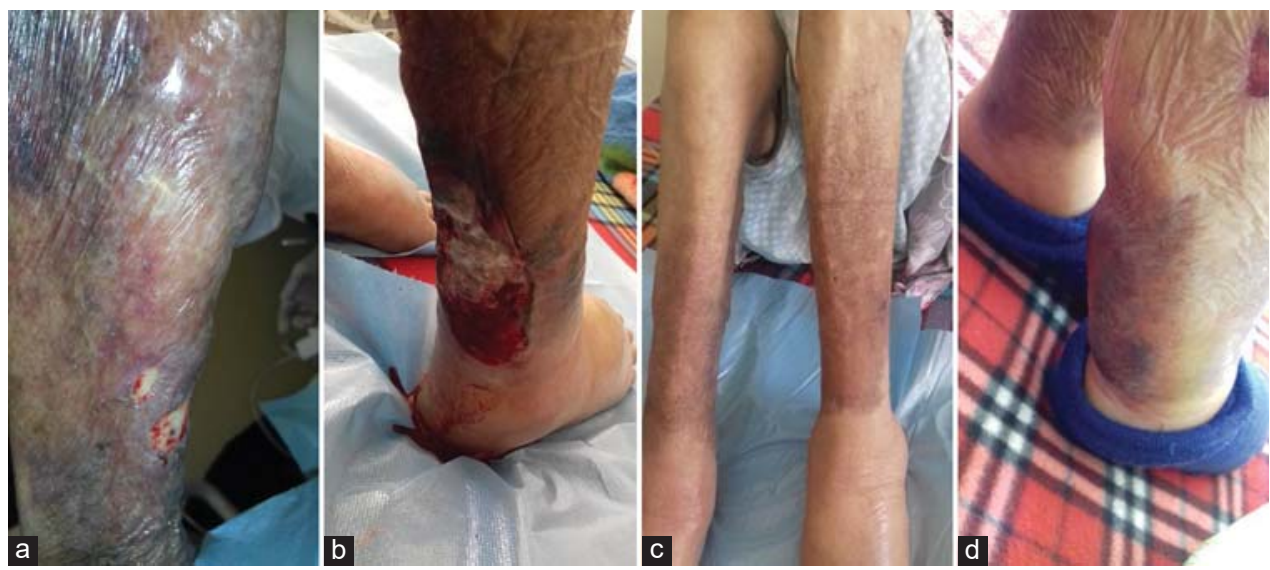

Figure 1: (a-d) well-defined violaceous plaques with erythematous margins and large areas of blistering on her legs, with surrounding hyperpigmentation.

manifestations emerge late and gradually progress to encephalopathy. The diagnosis of pellagra is clinical. Niacin test is not essential for diagnosis and should not delay management [4]. Treatment essentially consists of correcting predisposing and vitamin supplementation. Nicotinamide is preferred to nicotinic acid. The prescription of poly-vitamin complexes is often necessary since multiple vitamin deficiencies are most often involved. Supplementation leads to a rapid regression of cutaneous, then neurological and digestive signs. Etiological treatment must also be undertaken: stopping the drug responsible, stopping the alcoholism or correcting a deficiency [5].

\section{CONCLUSION}

We rarely think about deficiency of niacin in our clinical practice. However, as cutaneous symptoms are the first to appear, it seems important to come across this affection before any aggravation, especially, neurological ones.

\section{Consent}

The examination of the patient was conducted according to the Declaration of Helsinki principles.
The authors certify that they have obtained all appropriate patient consent forms. In the form the patient(s) has/have given his/her/their consent for his/her/their images and other clinical information to be reported in the journal. The patients understand that their names and initials will not be published and due efforts will be made to conceal their identity, but anonymity cannot be guaranteed.

\section{REFERENCES}

1. Harsha N S, Suraj B M, Kanakavidu SS, Kodali R. Pellagra: A forgotten ailment in current clinical practice. Med J DY Patil Vidyapeeth. 2019;12:78-80.

2. Thornton A. M and Drummond C. J. An unexpected case of pellagra. Med J Aust. 2014;200:546-8.

3. Sharma B, Bakki S.R, Jain R, Dubey P, and Prakash S. A rare case of alcoholic pellagra encephalopathy with startle myoclonus and marked response to niacin therapy: time for a new dictum? BMJ Case Rep. 2013;2013:bcr2013008906.

4. Chabchoub I, Souissi A, Alaoui FZ, Saad S, Mokni M. La pellagre : un diagnostic à ne pas méconnaître. Rev Méd Inter. 2019;40:A114.

5. Piqué-Duran E, Pérez-Cejudo JA, Cameselle D, Palacios-Llopis S, García-Vázquez O. Pellagra: A clinical, histopathological and epidemiological study of 7 cases. 2012;103:51-8.

Copyright by Sara Dahhouki, et al. This is an open access article distributed under the terms of the Creative Commons Attribution License, which permits unrestricted use, distribution, and reproduction in any medium, provided the original author and source are credited.

Source of Support: Nil, Conflict of Interest: None declared. 